\title{
SIMPLE EXCEPTIONAL 16-DIMENSIONAL JORDAN TRIPLE SYSTEMS
}

\author{
THOMAS J. E. SCHWARZ
}

\begin{abstract}
We prove that every simple exceptional 16-dimensional Jordan triple system is a subtriple of an exceptional Jordan algebra.
\end{abstract}

We prove a reverse triality principle which, in the language of Jordan pairs, states that every (semi)linear automorphism of a 16-dimensional exceptional Jordan pair can be extended to an automorphism of the 27-dimensional Jordan pair of the exceptional reduced Jordan algebra $H_{3}(\theta), \theta$ an octonion algebra. As an immediate application, we obtain that every 16-dimensional exceptional Jordan triple system is a subtriple of a 27-dimensional exceptional Jordan triple system. We will use the notations of [2] for Jordan pairs and those of $[\mathbf{1}, \mathbf{2}]$ for the exceptional reduced Jordan algebra. We make no assumption about our base field $F$. A similar, but different, situation has been examined in [4].

We recall some definitions: Let $\theta$ be an octonion (Cayley-Dickson) algebra over $F$. We have an involution $a \rightarrow \bar{a}, \operatorname{trace} \operatorname{tr}(a)=a+\bar{a}$, and norm $n(a)=a \bar{a}, a \in \theta$. Let $H_{3}(\theta)$ be the set of 3 by 3 matrices with entries in $\theta$ that are symmetric under $X \rightarrow{ }^{t} \bar{X}$ and have main diagonal entries in $F$. Every $X \in H_{3}(\theta)$ has the form

$$
\sum_{i=1}^{3} \alpha_{i}[i i]+\sum_{(i, j, k) \text { cyclic }} a_{i}[j k] \quad \text { with } \alpha_{i} \in F, a_{i} \in \theta,
$$

where $(i, j, k)$ is a cyclic permutation of $(1,2,3), a[i j]=a E_{i j}+\bar{a} E_{i j}, \alpha[i i]=\alpha E_{i i}$, in terms of the matrix units $E_{i j} . H_{3}(\theta)$ is a quadratic Jordan algebra with norm

$$
\begin{aligned}
& N\left(\sum \alpha_{i}[i i]+\sum a_{i}[j k]\right) \\
& \quad=\alpha_{1} \alpha_{2} \alpha_{3}-\alpha_{1} n\left(a_{1}\right)-\alpha_{2} n\left(a_{2}\right)-\alpha_{3} n\left(a_{3}\right)+\operatorname{tr}\left(a_{1} a_{2} a_{3}\right) .
\end{aligned}
$$

Two copies of $H_{3}(\theta)$ define the associated Jordan pair $\underline{V}$. A vector space semiautomorphism $\eta: H_{3}(\theta) \rightarrow H_{3}(\theta)$ is the plus part of a Jordan pair automorphism of $\underline{V}$ iff it is a semisimilarity of $H_{3}(\theta)$, i.e., fulfills

$$
N(\eta(X))=p \tau(N(X))
$$

with $p \in F^{*}, \tau \in \operatorname{Gal}(F)$.

An isomorphism $\phi: \underline{V} \rightarrow \underline{V}^{\text {op }}$ with $\phi^{2}=$ id is called involution of $\underline{V}$. ( $V^{\text {op }}$ is the Jordan pair with plus and minus parts exchanged.) An involution of $\underline{V}$ (up to conjugacy by an automorphism) corresponds to a Jordan triple structure (up to isomorphy) of $\underline{V}[3,1.13 ; 6]$.

Received by the editors February 26, 1986 and, in revised form June 19, 1986.

1980 Mathematics Subject Classification (1985 Revision). Primary 17C99. 
Let $E_{1}$ denote $(1[11], 1[11]) \in \underline{V}=\left(H_{3}(\theta), H_{3}(\theta)\right.$. The Jordan pair $\left[M_{1,2}(\theta)\right.$, $\left.M_{2,1}(\theta)\right]$ is isomorphic to the Peirce 1-space $\underline{V}_{1}\left(E_{1}\right)$ of $\underline{V}$, where $\underline{V}\left(E_{1}\right)^{\sigma}=\left\{a_{2}[31]\right.$ $\left.+a_{3}[12] \mid a_{2}, a_{3} \in \theta\right\}, \sigma= \pm 1$.

Our main result is the following theorem.

THEOREM. Every Jordan pair automorphism $\Phi=\left(\Phi^{+}, \Phi^{-}\right)$of $\underline{V}_{1}\left(E_{1}\right)$ can be extended to a Jordan pair automorphism of $\underline{V}$ fixing $E_{1}$.

Of course, every automorphism of $\underline{V}$ which leaves $\underline{V}_{2}\left(E_{1}\right)$ invariant, also leaves $V_{1}\left(E_{1}\right)$ invariant.

PROOF. A quasi-invertible element $(x, y)$ of $\underline{V}_{1}\left(E_{1}\right)$ remains quasi-invertible in $\underline{V}$, and the corresponding inner automorphism $\beta(x, y)$ of $\underline{V}$ maps $E_{1}$ to an idempotent in $V_{2}\left(E_{1}\right)$ which necessarily is of the form $\left(\alpha[11], \alpha^{-1}[11]\right)$.

If $\theta$ is not split $(1[31], 1[31])$ is a frame of $V_{1}\left(E_{2}\right)$. Otherwise there exist two orthogonal idempotents $e_{1}, e_{2}$ of $\theta$ such that $e_{1}+e_{2}=1$, in which case $\left(\left(e_{1}[31], e_{1}[31]\right)\right.$, $\left.\left(e_{2}[31], e_{2}[31]\right)\right)$ is a frame of $V_{1}\left(E_{1}\right)$.

Petersson's Conjugacy Theorem [5] asserts that there exist an inner automorphism of $V_{1}\left(E_{1}\right)$ which maps the image under $\Phi$ of this frame to the frame itself. As inner automorphisms can be lifted we only have to follow $\Phi$ by this inner automorphism to be able to assume $\Phi^{\sigma}(1[31])=1[31]$. Then $\Phi$ leaves invariant the Peirce decomposition of $\underline{V}_{1}\left(E_{1}\right)$ with respect to $(1[31], 1[31])$. We define coordinate maps by

We define

$$
\phi_{12}^{\sigma}(x)[12]=\Phi^{\sigma}(x[12]), \quad \phi_{31}^{\sigma}(y)[31]=\Phi^{\sigma}(y[31]) .
$$

$$
p^{\sigma}=\phi_{12}^{\sigma}(1) \in \theta^{*} .
$$

We use the Jordan matrix identities

(JM1) $Q_{x[i j]}^{\sigma} y[i j]=x \bar{y} x[i j]$,

(JM2) $\{x[i j], y[j i], z[i k]\}=x(y x)[i k]$

for $(i, j),(i, k)=(1,2),(3,1)$ to obtain

$$
\phi_{i j}^{\sigma}(x \bar{y} x)=\phi_{i j}^{\sigma}(x) \overline{\phi_{i j}^{-\sigma}(y)} \phi_{i j}^{\sigma}(x),
$$

where $(i, j)$ is either $(1,2)$ or $(3,1)$,

$$
\begin{aligned}
& \phi_{12}^{\sigma}(x)\left(\overline{\phi_{12}^{-\sigma}(y)} \overline{\phi_{31}^{\sigma}(z)}\right)=\overline{\left.\phi_{31}^{\sigma}((z y) \bar{x})\right)}, \\
& \overline{\phi_{31}^{\sigma}(x)}\left(\phi_{31}^{-\sigma}(y) \phi_{12}^{\sigma}(z)\right)=\phi_{12}^{\sigma}(\bar{x}(y z)) .
\end{aligned}
$$

Let us abbreviate $\phi_{31}^{\sigma}$ by $\phi^{\sigma}$. Recall $\phi^{\sigma}(1)=1$. A consequence of (1) is

$$
\phi^{-\sigma}(x)=\overline{\phi^{\sigma}(\bar{x})} \text { and } p^{\sigma} \overline{p^{-\sigma}}=1 \text {. }
$$

Setting $x=z=1$ in (3) yields

$$
\phi^{-\sigma}(x) p^{\sigma}=\phi_{12}^{\sigma}(x) .
$$

If we let $z=1$ in (3), using (4) and (5), we obtain the crucial identity

$$
\phi^{-\sigma}(x)\left(\phi^{-\sigma}(y) p^{\sigma}\right)=\phi^{-\sigma}(x y) p^{\sigma} .
$$

We let $z=1$ in (2) to get

$$
\phi^{\sigma}(x y)=\left(\phi^{\sigma}(x) p^{-\sigma}\right)\left(\overline{p^{\sigma}} \phi^{\sigma}(y)\right) .
$$


If (6) holds for one $\sigma$, we can use (4) and (5) to define an automorphism of $\underline{V}_{1}\left(E_{1}\right)$. Indeed, the identity $(a c)(\bar{c} b)=(a(b c)) \bar{c}$ for octonion algebras implies that $(6)$ and (7) are equivalent. This enables us to show by a straightforward calculation that (6) for one $\sigma$ implies (6) for $-\sigma$. By using the octonion identity $(a(b c))\left(c^{-1} a\right)=a b a$ we may prove that (6) implies (1), (2), and (3). (Both octonion identities may be proved by explicit calculation in the Cayley-Dickson algebra.)

Not let us assume that $\Phi$ is $\tau$-linear, $\tau \in \operatorname{Aut}(F)$. We extend $\Phi$ by defining

$$
\begin{aligned}
& \Phi^{\sigma}(x[11])=\tau(x)[11], \quad \Phi^{\sigma}(x[22])=\tau(x) n\left(p^{\sigma}\right)[22], \\
& \Phi^{\sigma}(x[33])=\tau(x)[33], \quad \Phi^{\sigma}(x[23])=\overline{p^{\sigma}} \phi^{-\sigma}(x)[23] \text {. }
\end{aligned}
$$

In order to show that $\Phi$ is an automorphism of $\underline{V}$, it suffices to show that $\Phi^{\sigma}$ is an element of the structure group of $H_{3}(\theta)[3,1.8]$ or equivalently a semisimilarity of $H_{3}(\theta)[1$, p. 10$]$, i.e.

$$
N\left(\Phi^{\sigma}(X)\right)=n\left(p^{\sigma}\right) \tau(N(X)) \quad \text { for } X \in H_{3}(\theta) .
$$

In order to prove this we first note that (1) implies $\phi^{\sigma}\left(x^{2}\right)=\left(\phi^{\sigma}(x)\right)^{2}$; hence,

$$
\operatorname{tr}\left(\phi^{\sigma}(x)\right) \phi^{\sigma}(x)-n\left(\phi^{\sigma}(x)\right)=\phi^{\sigma}(\operatorname{tr}(x) x-n(x))=\tau(\operatorname{tr}(x)) \phi^{\sigma}(x)-\tau(n(x)) .
$$

This implies $\operatorname{tr}\left(\phi^{\sigma}(x)\right)=\tau(\operatorname{tr}(x))$ and $\tau(n(x))=n\left(\phi^{\sigma}(x)\right)$. Therefore we have only to show

$$
\operatorname{tr}\left[\left(\overline{p^{\sigma}} \phi^{-\sigma}\left(a_{1}\right)\right)\left(\phi^{\sigma}\left(a_{2}\right)\right)\left(\phi^{-\sigma}\left(a_{3}\right) p^{\sigma}\right)\right]=n\left(p^{\sigma}\right) \tau\left(\operatorname{tr}\left(a_{1} a_{2} a_{3}\right)\right) .
$$

This is proven by the following calculation, in which we use the linearity as well as the associativity of the trace, which states that $\operatorname{tr}(a(b c))=\operatorname{tr}((a b) c)$ for $a, b, c \in \theta$ :

$$
\begin{aligned}
& \operatorname{tr}\left[\left(\overline{p^{\sigma}} \phi^{-\sigma}\left(a_{1}\right)\right)\left(\phi^{\sigma}\left(a_{2}\right)\right)\left(\phi^{-\sigma}\left(a_{3}\right) p^{\sigma}\right)\right] \\
&=\operatorname{tr}\left[\left(\overline{p^{\sigma}} \phi^{-\sigma}\left(a_{1}\right)\right)\left(\operatorname{tr}\left(\phi^{\sigma}\left(a_{2}\right)\right)-\overline{\phi^{\sigma}\left(a_{2}\right)}\right)\left(\phi^{-\sigma}\left(a_{3}\right) p^{\sigma}\right)\right] \\
&=\operatorname{tr}\left[\left(\overline{p^{\sigma}} \phi^{-\sigma}\left(a_{1}\right)\right)\left(\operatorname{tr}\left(\phi^{\sigma}\left(a_{2}\right)\right)\right)\left(\phi^{-\sigma}\left(a_{3}\right) p^{\sigma}\right)\right] \\
&-\operatorname{tr}\left[\left(\overline{p^{\sigma}} \phi^{-\phi}\left(a_{1}\right)\right) \overline{\left(\phi^{\sigma}\left(a_{2}\right)\right)}\left(\phi^{-\sigma}\left(a_{3}\right) p^{\sigma}\right)\right] \\
&= \operatorname{tr}\left[\phi^{\sigma}\left(a_{2}\right)\right] \operatorname{tr}\left[\left(\overline{p^{\sigma}} \phi^{-\sigma}\left(a_{1}\right)\right)\left(\phi^{-\sigma}\left(a_{3}\right) p^{\sigma}\right)\right] \\
&-\operatorname{tr}\left[\left(\overline{p^{\sigma}} \phi^{-\sigma}\left(a_{1}\right)\right)\left(\phi^{-\sigma}\left(\overline{a_{2}}\right)\right)\left(\phi^{-\sigma}\left(a_{3}\right) p^{\sigma}\right)\right] \quad \text { by }(4) \\
&= \operatorname{tr}\left[\phi^{\sigma}\left(a_{2}\right)\right] \operatorname{tr}\left[\overline{\left.\left(\overline{p^{\sigma}} \phi^{-\sigma}\left(a_{1}\right)\right)\left(\phi^{-\sigma}\left(a_{3}\right) p^{\sigma}\right)\right]}\right. \\
&-\operatorname{tr}\left[\left(\overline{p^{\sigma}} \phi^{-\sigma}\left(a_{1}\right)\right)\left(\phi^{-\sigma}\left(\overline{a_{2}} a_{3}\right) p^{\sigma}\right)\right] \quad \text { by }(6) \\
&= \operatorname{tr}\left[\phi^{\sigma}\left(a_{2}\right)\right] n\left(p^{\sigma}\right) \operatorname{tr}\left[\left(\phi^{-\sigma}\left(a_{3}\right) p^{\sigma}\right)\left(\overline{p^{-\sigma}} \phi^{-\sigma}\left(a_{1}\right)\right)\right] \\
&-n\left(p^{\sigma}\right) \operatorname{tr}\left[\left(\overline{p^{\sigma}} \phi^{-\sigma}\left(a_{1}\right)\right)\left(\phi^{-\sigma}\left(\overline{a_{2}} a_{3}\right) p^{-\sigma}\right)\right] \quad \text { by }(4) \\
&= n\left(p^{\sigma}\right) \operatorname{tr}\left[\phi^{\sigma}\left(a_{2}\right)\right] \operatorname{tr}\left[\phi^{-\sigma}\left(a_{3} a_{1}\right)\right]-n\left(p^{\sigma}\right) \operatorname{tr}\left[\phi^{-\sigma}\left(a_{1}\left(\overline{a_{2} a_{3}}\right)\right)\right] \\
&= n\left(p^{\sigma}\right) \tau\left(\operatorname{tr}\left(a_{2}\right) \operatorname{tr}\left(a_{3} a_{1}\right)\right)-n\left(p^{\sigma}\right)\left(\tau\left(\operatorname{tr}\left(a_{1} a_{2} a_{3}\right)\right)\right) \\
&= n\left(p^{\sigma}\right) \tau\left(\operatorname{tr}\left(a_{3} \operatorname{tr}\left(a_{2}\right) a_{1}\right)-\operatorname{tr}\left(a_{1} \overline{a_{2}} a_{3}\right)\right) \\
&= n\left(p^{\sigma}\right) \tau\left(\operatorname{tr}\left(a_{1} a_{2} a_{3}\right)\right) .
\end{aligned}
$$

It is easy to show that a Jordan pair automorphism which is the identity on $\underline{V}_{2}\left(E_{1}\right)$ and $\underline{V}_{1}\left(E_{1}\right)$ is the identity on $\underline{V}$ and that the identity on $\underline{V}_{1}\left(E_{1}\right)$ can be lifted only 
to an automorphism given by

$$
\begin{aligned}
& \Phi^{\sigma}\left(\sum \alpha_{i}[i i]+\sum_{(i, j, k) \text { cyclic }} a_{i}[j k]\right) \\
& \quad=\alpha_{\sigma} \alpha_{1}[11]+\alpha_{-\sigma} \alpha_{2}[22]+\alpha_{-\sigma} \alpha_{3}[33]+\alpha_{-\sigma} a_{1}[23]+a_{2}[31]+a_{3}[12]
\end{aligned}
$$

where $\alpha_{\sigma} \in F, \alpha_{-\sigma}=\alpha_{\sigma}^{-1}$. This remark concludes the proof of the theorem.

As a consequence of the proof, we note that for linear $\Phi,(7)$ implies that $\phi^{\sigma}$ is an element of the structure group of the alternative algebra $\theta$. At the same time, it is an element of the automorphism group of the Jordan algebra $\theta^{(+)}$, and hence an element of $O^{1}(n)$, the rotation group of $n$. Every element of $O^{1}(n)$ can be written as

$$
U_{a_{1}} \circ J \circ \cdots \circ U_{a_{2 r}} \circ J
$$

where $J(x)=\bar{x}, n\left(a_{1}\right) \cdots n\left(a_{2 r}\right)=1, U_{a}(x)=a x a$, and can be extended by

$$
Q_{[22]+a_{1}[31]}^{+\sigma} \circ \cdots \circ Q_{[22]+a_{2 r}[31]}^{-\sigma}
$$

to a Jordan pair automorphism (see [1, Chapter 2]). This remark leads to a shorter proof of our result for linear automorphisms.

We apply our result to Jordan triple systems.

COROLLARY 1. Every involution of $\underline{V}_{1}\left(E_{1}\right)$ can be extended to an involution of $\underline{V}$.

PROOF. Let $\varepsilon$ denote the switching involution of $\underline{V}$. If $\eta$ is an involution of $\underline{V}_{1}\left(E_{1}\right)$, then $\varepsilon \circ \eta$ is an automorphism of $\underline{V}_{1}\left(E_{1}\right)$ which can be extended to an automorphism $\varepsilon \circ \hat{\eta}$ which is the identity on $\underline{V}_{2}\left(E_{1}\right)$. As $\eta^{2}=\mathrm{id},(\eta \circ \varepsilon) \circ(\varepsilon \circ \eta)=\mathrm{id}$, hence $(\hat{\eta} \circ \varepsilon)$ is the identity on $\underline{V}_{2}\left(E_{1}\right)$ and $\underline{V}_{1}\left(E_{1}\right)$. Therefore $\hat{\eta}^{2}=$ id.

COROLlARY 2. Every simple exceptional 16-dimensional Jordan triple system is a Jordan subtriple system of an exceptional simple reduced Jordan algebra.

ProOF. A simple exceptional 16-dimensional Jordan triple system is given by an involution of a simple exceptional 16-dimensional Jordan pair.

\section{REFERENCES}

1. J. Faulkner, Octonion planes defined by quadratic Jordan algebras, Mem. Amer. Math. Soc. No. 104 (1970).

2. N. Jacobson, Structure theory of Jordan algebras, The University of Arkansas Lecture Notes in Math., vol. 5, Fayetteville, 1981.

3. O. Loos, Lecture Notes in Math., vol. 460, Springer-Verlag, Berlin and New York, 1975.

4. E. Neher, Klassifikation der einfachen reellen Ausnahme-Jordan-Tripelsysteme, J. Reine Angew. Math. 322 (1981), 145-169.

5. H. Petersson, Conjugacy of idempotents in Jordan pairs, Comm. Algebra 6 (1978), 673-715.

6. T. Schwarz, Special simple Jordan triple systems, Algebras Groups Geom. 2 (1985), 117-128. 1101

Department of Mathematics, Ohio State University, Columbus, Ohio 4321093309

Current address: Department of Mathematics, California State College, Bakersfield, California 